\title{
Spirodiclofen Analogues as Potential Lipid Biosynthesis Inhibitors: A Convenient Synthesis, Biological Evaluation, and Structure-Activity Relationship
}

\author{
Shaoyong Ke, 'Tingting Sun, Zhigang Zhang, Ya-Ni Zhang, Ying Liang, Kaimei Wang, and Ziwen Yang \\ Hubei Biopesticide Engineering Research Center, Hubei Academy of Agricultural Sciences, Wuhan 430064, P. R. China \\ ${ }^{*}$ E-Mail: keshaoyong@163.com \\ Received February 8, 2010, Accepted June 30, 2010
}

\begin{abstract}
Twenty spirodiclofen analogues have been designed and conveniently synthesized via three steps including esterification, one-pot heterocyclization, and acylation reactions. The target molecules have been identified on the basis of analytical spectra ( ${ }^{1} \mathrm{H}$ NMR, ${ }^{13} \mathrm{C}$ NMR and ESI-MS) data. All newly synthesized compounds have been screened for their potential insecticidal and herbicidal activity by standard method. The preliminary assays indicated that some of analogues displayed moderate to good insecticidal activity against Plutella xylostella compared with spirodiclofen, and some compounds showed obvious activity against Brassica chinensis. Structure-activity relationship (SAR) is also discussed based on the experimental data.
\end{abstract}

Key Words: Spirodiclofen analogues, Synthesis, Biological activity, Structure-activity relationship

\section{Introduction}

Highly efficacious acaricides/insecticides with novel modes of action are becoming increasingly important during the course of integrated pest management (IPM) strategy. ${ }^{1,2}$ Nowadays, since mites usually acquire resistance rapidly, the useful life of acaricides on the market is becoming shorter. ${ }^{3}$ Thus the focus of novel acaricides is shifting towards more effective, eco-friendly new structures with novel modes of action. Recently, spirodiclofen (Trade name: Envidor ${ }^{\circledR}$, and Daniemon ${ }^{\circledR}$; Figure 1) derived from natural tetronic acid core, a novel broad spectrum, non-systemic acaricide, was discovered by Bayer CropScien$\mathrm{ce},{ }^{4-6}$ which belong to the novel chemical class of spirocyclic phenyl-substituted tetronic acid derivatives. Spirodiclofen controls a broad range of phytophagous mite species such as Tetranychus, Phyllocoptruta, Panonychus, etc. at all stages of growth and has a unique mode of action differed from other acaricides, ${ }^{7,8}$ which interferes the lipid biosynthesis and blocks the enzyme acetyl-coenzyme A carboxylase that allows mites to form important fatty acids. ${ }^{9-12}$ Bayer Company has already developed the three tetronic acids analogues as acaricides and insecticides named spirodiclofen, ${ }^{5,6}$ spiromesifen, ${ }^{13-16}$ and spirotetramat (Figure 1) ${ }^{17-21}$ respectively.

However, both the mode of action and the structure of receptor for these compounds are not yet sure, and the systemic and detailed structure-activity relationships are unreported,

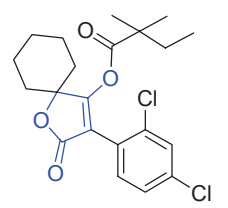

Spirodiclofen

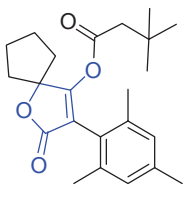

Spiromesifen

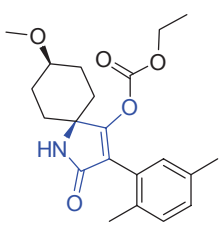

Spirotetramat
Figure 1. Structures of novel insecticides/acaricides derived from natural tetronic acid. which restrict the creation and optimization for these kinds of novel acaricides to some extent. Owing to the unique natural keto-enol structure, novel mode of action and potential significance in insect resistance management ${ }^{22-26}$ for spirocyclic tetronic acid derivatives, so it is important to establish the structure-activity relationships and extend activity profile for these compounds.

The aim of the present study was to develop more effective or broad-spectrum bioactive derivatives of spirodiclofen that could serve as potential lipid biosynthesis inhibitors for agrochemicals and explore the preliminary structure-activity relationships for these derivatives. As part of our agrochemistry program aimed at the search for novel heterocycle-based bioactive molecules, we wish to report herein the convenient synthesis, and biological evaluation of series of spiro-tetronic acid derivatives (Figure 2).

\section{Experimental}

Instrumentation and chemicals. All melting points ( $\mathrm{mp}$ ) were obtained using a digital model X-5 apparatus and are uncorrected. ${ }^{1} \mathrm{H}$ NMR and ${ }^{13} \mathrm{C}$ NMR spectra were recorded on a Brucker AM-400 (400 MHz) spectrometer with $\mathrm{CDCl}_{3}$ or DMSO- $d_{6}$ as the solvent and TMS as the internal standard. Chemical shifts are reported in $\delta$ (parts per million) values. Coupling constants ${ }^{\mathrm{n}} J$ are reported in Hz. Mass spectra were performed on a Micro-

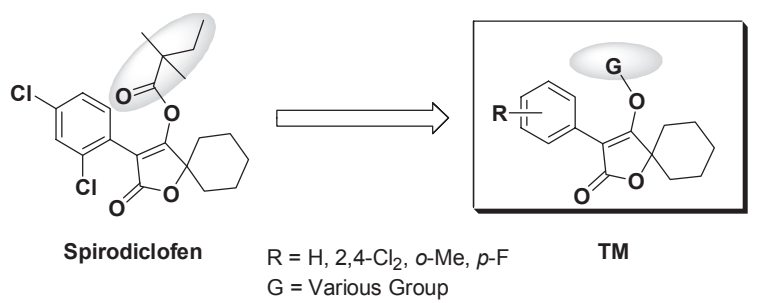

Figure 2. Design strategy of the target compounds. 
Mass Quattro micro ${ }^{\mathrm{TM}}$ API instrument. Analytical thin-layer chromatography (TLC) was carried out on precoated plates (silica gel 60 F254), and spots were visualized with ultraviolet (UV) light. All chemicals or reagents were purchased from standard commercial supplies. Anhydrous $\mathrm{CH}_{2} \mathrm{Cl}_{2}$ and THF were prepared by standard methods. All other solvents and reagents were analytical reagent and used directly without purification.

General synthetic procedure for the intermediates esters 2 and 4. The various esters $\mathbf{2}$ and $\mathbf{4}$ were prepared via normal esterification reactions of an acid with methanol under catalytic amount of sulfuric acid. Following the brief descriptions: the various acids $(0.10 \mathrm{~mol})$ is dissolved in $50 \mathrm{~mL}$ anhydrous methanol, $3 \mathrm{~mL}$ of conc. sulfuric acid is added, and the mixture refluxed for $5-8 \mathrm{~h}$. Part of the methanol is distilled in vacuo and the residue is poured into $80 \mathrm{~mL}$ of ice water. The mixture is extracted three times with $30 \mathrm{~mL}$ of ethyl acetate. The combined extracts are washed with $30 \mathrm{~mL}$ of water, dried over anhydrous sodium sulfate, evaporated in vacuo to obtain the residue, which was used for the next step reaction without further purification.

General synthetic procedure for the tetronic acid 5a-d. The important intermediate tetronic acid $\mathbf{5 a - d}$ is conveniently obtained by the modified one-pot cyclization method developed by Thierry Le Gall et al. ${ }^{27,28}$ The modified synthetic method are as follows: To a solution of substituted phenylacetate $(0.01$ mol, 1 equiv) and methyl 1-hydroxycyclohexanecarboxylate $1.90 \mathrm{~g}$ ( $0.012 \mathrm{~mol}, 1.2$ equiv) in anhydrous THF was added a solution of potassium tert-butoxide $2.46 \mathrm{~g}(0.022 \mathrm{~mol}, 2.2$ equiv $)$ in THF. The suspension obtained was then refluxed under nitrogen overnight. After cooling to ambient temperature, the reaction mixture was acidified with $5 \% \mathrm{HCl}$ aqueous to $\mathrm{pH} 2-3$, and then was extracted three times with ethyl acetate. The combined organic layers were then washed with water and dried over $\mathrm{Na}_{2} \mathrm{SO}_{4}$. After filtration and concentration under vacuum, the residue was taken up into little ethyl acetate $(3-5 \mathrm{~mL})$ and filtered in vacuo to give the corresponding tetronic acid 5a-d as white powder. Their physico-chemical properties and the spectra data are as follows:

4-Hydroxy-3-phenyl-1-oxaspiro[4.5] dec-3-en-2-one (5a): This compound was obtained following the above-described method as white powder, yield $76 \%, \mathrm{mp} 256-257{ }^{\circ} \mathrm{C} ;{ }^{1} \mathrm{H} \mathrm{NMR}$ $\left(400 \mathrm{MHz}, \mathrm{DMSO}-d_{6}\right) \delta 12.40$ (bs, $\left.1 \mathrm{H}, \mathrm{OH}\right), 7.21-7.87$ (m, 5H, $\mathrm{Ph}-\mathrm{H}), 1.19-2.06$ (m, 10H, Cyclohexyl-H); ESI-MS: calcd for $\mathrm{C}_{15} \mathrm{H}_{16} \mathrm{O}_{3}$ ([M+1] $\left.]^{+}\right), 245.3$; found, 245.8 .

4-Hydroxy-3-o-tolyl-1-oxaspiro[4.5] dec-3-en-2-one (5b): This compound was obtained following the above-described method as white powder, yield $78 \%, \operatorname{mp} 223-225{ }^{\circ} \mathrm{C} ;{ }^{1} \mathrm{H}$ NMR $\left(400 \mathrm{MHz}, \mathrm{DMSO}-d_{6}\right) \delta 12.08$ (bs, $\left.1 \mathrm{H}, \mathrm{OH}\right), 7.11-7.24$ (m, 4H, $\mathrm{Ph}-\mathrm{H}), 2.15$ (s, 3H, Ph-CH 3 ), 1.23-1.96 (m, 10H, Cyclohexyl-H); ESI-MS: calcd for $\mathrm{C}_{16} \mathrm{H}_{18} \mathrm{O}_{3}\left([\mathrm{M}+1]^{+}\right), 259.3$; found, 259.8 .

3-(2,4-Dichlorophenyl)-4-hydroxy-1-oxaspiro[4.5] dec-3en-2-one (5c): This compound was obtained following the above-described method as white powder, yield 80\%, mp 249 $250{ }^{\circ} \mathrm{C} ;{ }^{1} \mathrm{H}$ NMR (400 MHz, DMSO- $\left.d_{6}\right) \delta 12.46$ (bs, $\left.1 \mathrm{H}, \mathrm{OH}\right)$, 7.28-7.63 (m, 3H, Ph-H), 1.04-1.85 (m, 10H, Cyclohexyl-H); ESI-MS: calcd for $\mathrm{C}_{15} \mathrm{H}_{14} \mathrm{Cl}_{2} \mathrm{O}_{3}\left([\mathrm{M}+1]^{+}\right)$, 314.2; found, 313.8 .

3-(4-Fluorophenyl)-4-hydroxy-1-oxaspiro[4.5] dec-3-en-2one (5d): This compound was obtained following the abovedescribed method as white powder, yield $72 \%, \mathrm{mp}>260{ }^{\circ} \mathrm{C}$;
${ }^{1} \mathrm{H} \mathrm{NMR}\left(400 \mathrm{MHz}, \mathrm{DMSO}-d_{6}\right) \delta 12.45$ (bs, $\left.1 \mathrm{H}, \mathrm{OH}\right), 7.91-7.95$ (m, 2H, Ph-H), 7.21 (t, J=9 Hz, 2H, Ph-H), 1.19-2.05 (m, 10H, Cyclohexyl-H); ESI-MS: calcd for $\mathrm{C}_{15} \mathrm{H}_{15} \mathrm{FO}_{3}\left([\mathrm{M}+1]^{+}\right), 263.3$; found, 263.8.

General synthetic procedure for the target compounds 6a-t. The typical process of synthesis of spirodiclofen analogues 6a-t is shown as following: To a stirred solution of 4-hydroxy3-(substituted phenyl)-1-oxaspiro[4.5]dec-3-en-2-one 5a-d $(1 \mathrm{mmol})$ and $\mathrm{Et}_{3} \mathrm{~N}(1.5 \mathrm{mmol})$ in anhydrous dichloromethane $(10 \mathrm{~mL})$ under an ice bath, and various acyl chloride $(1.1 \mathrm{mmol})$ in dichloromethane $(5 \mathrm{~mL})$ was added dropwise. The mixture was stirred at low temperature for about $1 \mathrm{~h}$ and then was allowed to react at ambient temperature for another 1 - 4 hours, which was detected by TLC. After this, the reaction mixture was poured into water and extracted with dichloromethane. The combined organic layer was washed extensively with aqueous sodium bicarbonate, brine and water, and then dried over anhydrous sodium sulfate. The solvent was evaporated, and the residue was purified by column chromatography on silica gel using petroleum ether/ethyl acetate as eluent to afford the target compounds 6a-t. Their physico-chemical properties and the spectra data are as follows:

3-(2,4-Dichlorophenyl)-2-ox0-1-oxaspiro[4.5]dec-3-en-4yl methanesulfonate (6a): This compound was obtained following the above-described method as white solid, yield $81 \%$, mp $138-140{ }^{\circ} \mathrm{C} ;{ }^{1} \mathrm{H} \mathrm{NMR}\left(400 \mathrm{MHz}, \mathrm{CDCl}_{3}\right) \delta 7.49\left(\mathrm{~d},{ }^{4} \mathrm{~J}=1.2\right.$ $\mathrm{Hz}, 1 \mathrm{H}, \mathrm{Ph}-\mathrm{H}$ ), 7.34-7.39 (m, 2H, Ph-H), 2.99 (s, 3H, $\left.\mathrm{CH}_{3}-\mathrm{S}\right)$, 1.73-1.94 (m, 10H, Cyclohexyl-H); ${ }^{13} \mathrm{C}$ NMR (100 MHz, $\left.\mathrm{CDCl}_{3}\right)$ $\delta 169.68,168.28,136.41,134.59,132.87,129.58,127.57,125.58$, 84.59, 40.31, 33.10, 32.11, 32.09, 24.23, 21.62; ESI-MS: calcd for $\mathrm{C}_{16} \mathrm{H}_{16} \mathrm{Cl}_{2} \mathrm{O}_{5} \mathrm{~S}\left(\mathrm{M}^{+}\right)$, 391.3; found, 391.8 .

3-(2,4-Dichlorophenyl)-2-oxo-1-oxaspiro[4.5] dec-3-en-4yl ethyl carbonate (6b): This compound was obtained following the above-described method as white powder, yield $86 \%, \mathrm{mp}$ $79-80{ }^{\circ} \mathrm{C} ;{ }^{1} \mathrm{H} \mathrm{NMR}\left(400 \mathrm{MHz}, \mathrm{CDCl}_{3}\right) \delta 7.41\left(\mathrm{~d},{ }^{4} \mathrm{~J}=2 \mathrm{~Hz}, 1 \mathrm{H}\right.$, $\mathrm{Ph}-\mathrm{H}), 7.38$ (d, $J=8.4 \mathrm{~Hz}, 1 \mathrm{H}, \mathrm{Ph}-\mathrm{H}), 7.31\left(\mathrm{dd},{ }^{4} J=2 \mathrm{~Hz}, J=\right.$ $8 \mathrm{~Hz}, 1 \mathrm{H}, \mathrm{Ph}-\mathrm{H}), 4.15$ (q, $\left.J=7 \mathrm{~Hz}, 2 \mathrm{H}, \mathrm{CH}_{2} \mathrm{CH}_{3}\right), 1.72-1.91$ (m, 10H, Cyclohexyl-H), $1.24\left(\mathrm{t}, J=7 \mathrm{~Hz}, 3 \mathrm{H}, \mathrm{CH}_{2} \mathrm{CH}_{3}\right) ;{ }^{13} \mathrm{C}$ NMR $\left(100 \mathrm{MHz}, \mathrm{CDCl}_{3}\right) \delta 171.53,170.58,149.94,136.62$, 135.68, 133.07, 130.38, 128.48, 128.26, 111.42, 84.86, 67.66, 47.05, 34.15, 25.55, 22.91, 15.06, 9.80; ESI-MS: calcd for $\mathrm{C}_{18} \mathrm{H}_{18} \mathrm{Cl}_{2} \mathrm{O}_{5}\left([\mathrm{M}+1]^{+}\right), 386.2$; found, 385.8.

3-(2,4-Dichlorophenyl)-2-ox0-1-oxaspiro[4.5]dec-3-en-4yl 2-phenylacetate (6c): This compound was obtained following the above-described method as white crystal, yield $68 \%, \mathrm{mp}$ $85-86{ }^{\circ} \mathrm{C} ;{ }^{1} \mathrm{H}$ NMR $\left(400 \mathrm{MHz}, \mathrm{CDCl}_{3}\right) \delta$ 7.24-7.26 (m, 3H, Ph-H), 7.19 (s, 1H, Ph-H), 7.08-7.17 (m, 4H, Ph-H), 3.69 (s, 2H, $\left.\mathrm{CH}_{2} \mathrm{CO}\right), 1.53-1.70$ (m, 10H, Cyclohexyl-H); ${ }^{13} \mathrm{C}$ NMR (100 $\left.\mathrm{MHz}_{2} \mathrm{CDCl}_{3}\right) \delta 169.57,163.85,134.29,133.12,130.75,130.70$, $128.42,128.32$, 128.20, 128.16, 127.87, 127.65, 126.84, 126.24, 125.73, 111.13, 82.83, 39.83, 31.86, 23.35, 20.62; ESI-MS: calcd for $\mathrm{C}_{23} \mathrm{H}_{20} \mathrm{Cl}_{2} \mathrm{O}_{4}\left(\mathrm{M}^{+}\right), 431.3$; found, 431.9 .

3-(2,4-Dichlorophenyl)-2-oxo-1-oxaspiro[4.5] dec-3-en-4yl 2-(2,4-dichloro phenyl)acetate (6d): This compound was obtained following the above-described method as white flocculent solid, yield 88\%, mp $142-143{ }^{\circ} \mathrm{C} ;{ }^{1} \mathrm{H}$ NMR $(400 \mathrm{MHz}$, $\left.\mathrm{CDCl}_{3}\right) \delta 7.42$ (s, 1H, Ph-H), 7.37 (s, 1H, Ph-H), 7.28-7.33 (m, 2H, Ph-H), 7.22 (d, $J=8 \mathrm{~Hz}, 1 \mathrm{H}, \mathrm{Ph}-\mathrm{H}), 7.13$ (d, $J=8 \mathrm{~Hz}, 1 \mathrm{H}$, 
$\mathrm{Ph}-\mathrm{H}), 3.91$ (s, 2H, $\mathrm{CH}_{2} \mathrm{CO}$ ), 1.63-1.87 (m, 10H, Cyclohexyl-H); ${ }^{13} \mathrm{C} \mathrm{NMR}\left(100 \mathrm{MHz}, \mathrm{CDCl}_{3}\right) \delta 170.27,169.20,163.52,135.49$, $135.23,134.86,134.13,132.19,131.86,129.61,129.21,128.87$, $127.58,127.39,126.78,112.09,83.80,38.00,32.94,30.93$, 24.42, 21.68; ESI-MS: calcd for $\mathrm{C}_{23} \mathrm{H}_{18} \mathrm{Cl}_{4} \mathrm{O}_{4}\left([\mathrm{M}+1]^{+}\right), 501.2$; found, 501.5 .

3-(2,4-Dichlorophenyl)-2-oxo-1-oxaspiro[4.5] dec-3-en-4yl 2-o-tolylacetate (6e): This compound was obtained following the above-described method as yellow powder, yield 83\%, mp 102 - $104{ }^{\circ} \mathrm{C} ;{ }^{1} \mathrm{H}$ NMR $\left(400 \mathrm{MHz}, \mathrm{CDCl}_{3}\right) \delta 7.34\left(\mathrm{~d},{ }^{4} \mathrm{~J}=1.6\right.$ $\mathrm{Hz}, 1 \mathrm{H}, \mathrm{Ph}-\mathrm{H}), 7.20-7.24$ (m, 3H, Ph-H), 7.15 (t, $J=7.8 \mathrm{~Hz}, 2 \mathrm{H}$, Ph-H), 7.10 (d, $J=6.8$ Hz, 1H, Ph-H), 3.78 (s, 2H, $\mathrm{CH}_{2} \mathrm{CO}$ ), 2.17 (s, 3H, Ph- $\left.\mathrm{CH}_{3}\right), 1.59-1.79(\mathrm{~m}, 10 \mathrm{H}, \mathrm{Cyclohexyl}-\mathrm{H}) ;{ }^{13} \mathrm{C}$ NMR $\left(100 \mathrm{MHz}, \mathrm{CDCl}_{3}\right) \delta 170.64,169.26,164.63,136.79$, $135.33,134.18,131.77,130.66,130.45,130.24,129.21,128.19$, $127.29,126.84,126.44,112.16,83.80,38.62,32.89,24.40$, 21.67, 19.29; ESI-MS: calcd for $\mathrm{C}_{24} \mathrm{H}_{22} \mathrm{Cl}_{2} \mathrm{O}_{4}\left(\mathrm{M}^{+}\right)$, 445.3; found, 445.7.

3-(2,4-Dichlorophenyl)-2-oxo-1-oxaspiro[4.5] dec-3-en-4yl 3,4,5-trimethoxybenzoate (6f): This compound was obtained following the above-described method as white powder, yield $75 \%$, mp $183-185^{\circ} \mathrm{C} ;{ }^{1} \mathrm{H}$ NMR (400 MHz, $\left.\mathrm{CDCl}_{3}\right) \delta 7.44(\mathrm{~d}$, $J=8.4 \mathrm{~Hz}, 1 \mathrm{H}, \mathrm{Ph}-\mathrm{H}), 7.36\left(\mathrm{~d},{ }^{4} J=1.2 \mathrm{~Hz}, 1 \mathrm{H}, \mathrm{Ph}-\mathrm{H}\right), 7.32$ (dd, $J=8.4 \mathrm{~Hz}, 1 \mathrm{H}, \mathrm{Ph}-\mathrm{H}), 7.25$ (s, 2H, Ph-H), 3.94 (s, 3H, $\left.\mathrm{CH}_{3} \mathrm{O}\right), 3.90\left(\mathrm{~s}, 6 \mathrm{H}, \mathrm{CH}_{3} \mathrm{O}\right), 1.24-1.88$ (m, 10H, Cyclohexyl-H); ${ }^{13} \mathrm{C} \mathrm{NMR}\left(100 \mathrm{MHz}, \mathrm{CDCl}_{3}\right) \delta 170.80,169.40,159.84,153.17$, 144.02, 135.30, 134.20, 131.81, 129.31, 127.36, 127.02, 121.60, 112.00, 108.04, 83.93, 60.98, 56.39, 33.18, 24.39, 21.71; ESIMS: calcd for $\mathrm{C}_{25} \mathrm{H}_{24} \mathrm{Cl}_{2} \mathrm{O}_{7}\left(\mathrm{M}^{+}\right)$, 507.4; found, 507.9 .

3-(2,4-Dichlorophenyl)-2-oxo-1-oxaspiro[4.5] dec-3-en-4yl 4-fluorobenzoate (6g): This compound was obtained following the above-described method as white powder, yield $82 \%$, mp $155-156{ }^{\circ} \mathrm{C} ;{ }^{1} \mathrm{H}$ NMR $\left(400 \mathrm{MHz}, \mathrm{CDCl}_{3}\right) \delta 7.96-8.00(\mathrm{~m}$, 2H, Ph-H), 7.09-7.39 (m, 5H, Ph-H), 1.19-1.84 (m, 10H, Cyclohexyl-H); ${ }^{13} \mathrm{C}$ NMR $\left(100 \mathrm{MHz}, \mathrm{CDCl}_{3}\right) \delta 170.37,169.45$, 167.97, 165.41, 158.98, 135.30, 134.19, 133.36, 133.26, 131.80, 129.21, 127.34, 127.06, 123.33, 123.30, 116.40, 116.18, 111.58, 83.84, 33.12, 24.35, 21.69; ESI-MS: calcd for $\mathrm{C}_{22} \mathrm{H}_{17} \mathrm{Cl}_{2} \mathrm{FO}_{4}$ $\left([\mathrm{M}+1]^{+}\right), 436.3$; found, 435.9 .

2-Oxo-3-o-tolyl-1-oxaspiro[4.5] dec-3-en-4-yl 2,2-dimethylbutanoate (6h): This compound was obtained following the above-described method as white powder, yield $85 \%, \mathrm{mp} 87$ $89{ }^{\circ} \mathrm{C} ;{ }^{1} \mathrm{H}$ NMR (400 MHz, $\left.\mathrm{CDCl}_{3}\right) \delta$ 7.13-7.23 (m, 3H, Ph-H), 7.06 (d, $J=7.6 \mathrm{~Hz}, 1 \mathrm{H}, \mathrm{Ph}-\mathrm{H}), 2.27$ (s, 3H, $\left.\mathrm{Ph}-\mathrm{CH}_{3}\right), 1.71-1.85$ (m, 10H, Cyclohexyl-H), 1.50 (q, $J=7.6 \mathrm{~Hz}, 2 \mathrm{H}, \mathrm{CH}_{2} \mathrm{CH}_{3}$ ), $1.07\left(\mathrm{~s}, 6 \mathrm{H}, \mathrm{CH}_{3}\right), 0.62\left(\mathrm{t}, 3 \mathrm{H}, J=7.6 \mathrm{~Hz}, \mathrm{CH}_{2} \mathrm{CH}_{3}\right),{ }^{13} \mathrm{C} \mathrm{NMR}$ $\left(100 \mathrm{MHz}, \mathrm{CDCl}_{3}\right) \delta 172.24,171.39,169.83,137.27,130.88$, 130.31, 129.49, 129.00, 127.51, 125.61, 117.98, 84.00, 43.29, 33.26, 32.87, 30.92, 24.57, 24.38, 21.78, 19.57, 8.83; ESI-MS: calcd for $\mathrm{C}_{23} \mathrm{H}_{18} \mathrm{Cl}_{4} \mathrm{O}_{4}\left([\mathrm{M}+1]^{+}\right), 357.5$; found, 358.0.

2-Oxo-3-o-tolyl-1-oxaspiro[4.5] dec-3-en-4-yl methanesulfonate (6i): This compound was obtained following the abovedescribed method as colorless crystal, yield 94\%, mp 118 $121{ }^{\circ} \mathrm{C} ;{ }^{1} \mathrm{H} \mathrm{NMR}\left(400 \mathrm{MHz}, \mathrm{CDCl}_{3}\right) \delta$ 7.29-7.37 (m, 2H, Ph-H), 7.22-7.25 (m, 2H, Ph-H), 2.59 (s, 3H, $\left.\mathrm{CH}_{3}-\mathrm{S}\right), 2.30$ (s, 3H, Ph- $\left.\mathrm{CH}_{3}\right), 1.74-1.93$ (m, 10H, Cyclohexyl-H); ${ }^{13} \mathrm{C}$ NMR (100 $\left.\mathrm{MHz}, \mathrm{CDCl}_{3}\right) \delta 169.79,168.87,138.05,130.64,130.09,129.93$, $126.87,126.02$, 124.91, 118.80, 84.45, 40.46, 32.97, 32.48,
24.25, 21.68, 19.51; ESI-MS: calcd for $\mathrm{C}_{17} \mathrm{H}_{20} \mathrm{O}_{5} \mathrm{~S}\left([\mathrm{M}+1]^{+}\right)$, 337.4; found, 337.8 .

Ethyl 2-oxo-3-o-tolyl-1-oxaspiro[4.5]dec-3-en-4-yl carbonate (6j): This compound was obtained following the abovedescribed method as pale yellow solid, yield $74 \%, \mathrm{mp} 72-74{ }^{\circ} \mathrm{C}$; ${ }^{1} \mathrm{H}$ NMR (400 MHz, $\mathrm{CDCl}_{3}$ ) $\delta$ 7.07-7.19 (m, 4H, Ph-H), 3.94 (q, $\left.J=7.2 \mathrm{~Hz}, 2 \mathrm{H}, \mathrm{CH}_{2} \mathrm{CH}_{3}\right), 2.19$ (s, 3H, Ph-CH 3$), 1.65-1.82$ (m, 10H, Cyclohexyl-H), 1.03 (t, $\left.J=7.2 \mathrm{~Hz}, 3 \mathrm{H}, \mathrm{CH}_{2} \mathrm{CH}_{3}\right) ;{ }^{13} \mathrm{C}$ NMR $\left(100 \mathrm{MHz}, \mathrm{CDCl}_{3}\right) \delta 170.35,169.72,148.98,137.09$, 130.39, 129.25, 129.04, 127.27, 125.78, 115.74, 83.51, 66.14, 32.93, 24.33, 21.70, 19.54, 13.64; ESI-MS: calcd for $\mathrm{C}_{19} \mathrm{H}_{22} \mathrm{O}_{5}$ $\left([\mathrm{M}+1]^{+}\right), 331.4$; found, 331.9 .

2-Oxo-3-o-tolyl-1-oxaspiro[4.5] dec-3-en-4-yl 2-(2,4-dichlorophenyl)acetate (6k): This compound was obtained following the above-described method as white crystal, yield 90\%, mp $122-125^{\circ} \mathrm{C} ;{ }^{1} \mathrm{H}$ NMR (400 MHz, $\left.\mathrm{CDCl}_{3}\right) \delta 7.37$ (d, $J=2$ $\mathrm{Hz}, 1 \mathrm{H}, \mathrm{Ph}-\mathrm{H}), 7.03-7.26$ (m, 5H, Ph-H), 6.94 (d, J=8.4 Hz, $1 \mathrm{H}, \mathrm{Ph}-\mathrm{H}$ ), 3.77 (s, 2H, $\mathrm{CH}_{2} \mathrm{CO}$ ), 2.20 (s, 3H, Ph- $\mathrm{CH}_{3}$ ), 1.69$1.82\left(\mathrm{~m}, 10 \mathrm{H}\right.$, Cyclohexyl-H); ${ }^{13} \mathrm{C}$ NMR $\left(100 \mathrm{MHz}, \mathrm{CDCl}_{3}\right) \delta$ 206.92, 170.42, 169.41, 164.36, 137.15, 135.10, 134.58, 131.92, $130.39,129.47,129.21,129.04,128.97,127.45,127.25,125.74$, 118.16, 83.86, 50.75, 37.94, 33.06, 30.86, 24.42, 21.71, 19.48; ESI-MS: calcd for $\mathrm{C}_{24} \mathrm{H}_{22} \mathrm{Cl}_{2} \mathrm{O}_{4}\left(\mathrm{M}^{+}\right)$, 445.3; found, 445.9.

2-Oxo-3-o-tolyl-1-oxaspiro[4.5] dec-3-en-4-yl 2-o-tolylacetate (6I): This compound was obtained following the abovedescribed method as colorless crystal, yield 72\%, mp 103 $105{ }^{\circ} \mathrm{C} ;{ }^{1} \mathrm{H} \mathrm{NMR}\left(400 \mathrm{MHz}, \mathrm{CDCl}_{3}\right) \delta 6.99-7.23$ (m, 8H, Ph-H), 3.65 (s, 2H, $\mathrm{CH}_{2} \mathrm{CO}$ ), 2.14 (s, 3H, Ph- $\left.\mathrm{CH}_{3}\right), 1.99$ (s, 3H, Ph$\left.\mathrm{CH}_{3}\right), 1.61-1.78$ (m, 10H, Cyclohexyl-H) ${ }^{13} \mathrm{C}$ NMR (100 MHz, $\left.\mathrm{CDCl}_{3}\right) \delta 170.52,169.60,165.19,137.09,136.84,130.59$, 130.31, 130.11, 128.00, 127.39, 126.30, 125.72, 117.82, 83.76, 38.72, 32.94, 24.40, 21.70, 19.37, 19.04; ESI-MS: calcd for $\mathrm{C}_{25} \mathrm{H}_{26} \mathrm{O}_{4}\left([\mathrm{M}+1]^{+}\right), 391.5$; found, 391.9.

2-Oxo-3-o-tolyl-1-oxaspiro[4.5]dec-3-en-4-yl 3,4,5-trimethoxybenzoate (6m): This compound was obtained following the above-described method as white powder, yield $76 \%, \mathrm{mp}$ 170 - $172{ }^{\circ} \mathrm{C} ;{ }^{1} \mathrm{H} \mathrm{NMR}\left(400 \mathrm{MHz}, \mathrm{CDCl}_{3}\right) \delta 7.08-7.15$ (m, 6H, $\mathrm{Ph}-\mathrm{H}), 3.85$ (s, 3H, $\left.\mathrm{CH}_{3} \mathrm{O}\right), 3.81$ (s, 6H, $\left.\mathrm{CH}_{3} \mathrm{O}\right), 2.24$ (s, 3H, $\left.\mathrm{Ph}-\mathrm{CH}_{3}\right), 1.73-1.80$ (m, 10H, Cyclohexyl-H); ${ }^{13} \mathrm{C}$ NMR (100 $\left.\mathrm{MHz}, \mathrm{CDCl}_{3}\right) \delta 169.52,168.84,159.51,152.13,142.74,135.90$, 129.38, 128.24, 127.93, 126.63, 124.81, 120.93, 116.77, 106.77, 82.99, 59.99, 55.37, 32.33, 23.42, 20.80, 18.70; ESI-MS: calcd for $\mathrm{C}_{26} \mathrm{H}_{28} \mathrm{O}_{7}\left([\mathrm{M}+1]^{+}\right)$, 453.5; found, 454.0.

2-Oxo-3-o-tolyl-1-oxaspiro[4.5] dec-3-en-4-yl 4-fluorobenzoate (6n): This compound was obtained following the abovedescribed method as white powder, yield $90 \%, \mathrm{mp} 162-163{ }^{\circ} \mathrm{C}$; ${ }^{1} \mathrm{H} \mathrm{NMR}\left(400 \mathrm{MHz}, \mathrm{CDCl}_{3}\right) \delta$ 7.97-8.00 (m, 2H, Ph-H), 7.117.19 (m, 6H, Ph-H), 2.28 (s, 3H, $\left.\mathrm{CH}_{3}\right), 1.79-1.86(\mathrm{~m}, 10 \mathrm{H}$, Cyclohexyl-H); ${ }^{13} \mathrm{C}$ NMR (100 MHz, $\left.\mathrm{CDCl}_{3}\right) \delta 170.31,169.70$, 159.76, 136.89, 133.09, 133.00, 130.33, 129.18, 128.92, 127.54, $125.74,123.58,117.72,116.27,116.05,83.90,33.24,24.38$, 21.75, 19.62; ESI-MS: calcd for $\mathrm{C}_{23} \mathrm{H}_{21} \mathrm{FO}_{4}\left([\mathrm{M}+1]^{+}\right), 381.4$; found, 381.9 .

3-(4-Fluorophenyl)-2-oxo-1-oxaspiro [4.5] dec-3-en-4-yl 2,2-dimethylbutanoate (6o): This compound was obtained following the above-described method as white powder, yield $80 \%$, mp $98-99{ }^{\circ} \mathrm{C} ;{ }^{1} \mathrm{H} \mathrm{NMR}\left(400 \mathrm{MHz}, \mathrm{CDCl}_{3}\right) \delta 7.51-7.55$ (m, 2H, Ph-H), 7.07 (t, $J=8.6 \mathrm{~Hz}, 2 \mathrm{H}, \mathrm{Ph}-\mathrm{H}), 1.66-1.77$ (m, 
$11 \mathrm{H}$, Cyclohexyl- $\mathrm{H}$ and $\left.\mathrm{CH}_{2} \mathrm{CH}_{3}\right), 1.25\left(\mathrm{~s}, 6 \mathrm{H}, \mathrm{CH}_{3}\right), 0.87$ (t, $3 \mathrm{H}, J=7.4 \mathrm{~Hz}, \mathrm{CH}_{2} \mathrm{CH}_{3}$ ); ESI-MS: calcd for $\mathrm{C}_{21} \mathrm{H}_{25} \mathrm{FO}_{4}$ $\left([\mathrm{M}+1]^{+}\right), 361.4$; found, 361.9 .

3-(4-Fluorophenyl)-2-oxo-1-oxaspiro [4.5]dec-3-en-4-yl methanesulfonate (6p): This compound was obtained following the above-described method as white powder, yield $75 \%, \mathrm{mp}$ $111-112{ }^{\circ} \mathrm{C} ;{ }^{1} \mathrm{H} \mathrm{NMR}\left(400 \mathrm{MHz}, \mathrm{CDCl}_{3}\right) \delta 7.58-7.62(\mathrm{~m}, 2 \mathrm{H}$, $\mathrm{Ph}-\mathrm{H}), 7.14-7.18$ (m, 2H, Ph-H), 3.09 (s, 3H, $\left.\mathrm{CH}_{3}\right), 1.26-1.99$ (m, $10 \mathrm{H}$, Cyclohexyl-H); ESI-MS: calcd for $\mathrm{C}_{16} \mathrm{H}_{17} \mathrm{~F} \mathrm{O} 5 \mathrm{~S}\left([\mathrm{M}+1]^{+}\right)$, 341.4; found, 341.8 .

Ethyl 3-(4-fluorophenyl)-2-oxo-1-oxaspiro[4.5]dec-3-en-4yl carbonate (6q): This compound was obtained following the above-described method as white powder, yield $84 \%$, mp 187 $188{ }^{\circ} \mathrm{C} ;{ }^{1} \mathrm{H} \mathrm{NMR}\left(400 \mathrm{MHz}, \mathrm{CDCl}_{3}\right) \delta 8.02-8.06$ (m, 2H, Ph-H), 6.98-7.02 (m, 2H, Ph-H), 3.07 (q, $\left.J=7.4 \mathrm{~Hz}, 2 \mathrm{H}, \mathrm{CH}_{2} \mathrm{CH}_{3}\right)$, 1.57-2.14 (m, 10H, Cyclohexyl-H), 1.35 (t, $J=7 . \overline{4 \mathrm{~Hz}}, 3 \mathrm{H}$, $\left.\mathrm{CH}_{2} \mathrm{CH}_{3}\right)$; ESI-MS: calcd for $\mathrm{C}_{18} \mathrm{H}_{19} \mathrm{FO}_{5}\left([\mathrm{M}+1]^{+}\right), 335.3$; found, 335.9 .

2-Oxo-3-phenyl-1-oxaspiro[4.5] dec-3-en-4-yl 2,2-dimethylbutanoate (6r): This compound was obtained following the above-described method as white powder, yield 83\%, mp 105 $106{ }^{\circ} \mathrm{C} ;{ }^{1} \mathrm{H} \mathrm{NMR}\left(400 \mathrm{MHz}, \mathrm{CDCl}_{3}\right) \delta 7.26-7.46(\mathrm{~m}, 5 \mathrm{H}, \mathrm{Ph}-\mathrm{H})$, 1.56-1.75 (m, 12H, Cyclohexyl-H and $\left.\mathrm{CH}_{2} \mathrm{CH}_{3}\right), 1.17$ (s, 6H, $\left.\mathrm{CH}_{3}\right), 0.79$ (t, $\left.3 \mathrm{H}, J=7.4 \mathrm{~Hz}, \mathrm{CH}_{2} \mathrm{CH}_{3}\right) ;{ }^{13} \mathrm{C} \mathrm{NMR}(100 \mathrm{MHz}$, $\left.\mathrm{CDCl}_{3}\right) \delta 171.35,169.03,127.82,127.40,127.20,127.10$, 115.54, 82.63, 42.32, 32.16, 31.79, 23.58, 23.42, 20.73, 8.09; ESI-MS: calcd for $\mathrm{C}_{21} \mathrm{H}_{26} \mathrm{O}_{4}\left([\mathrm{M}+1]^{+}\right), 343.4$; found, 343.9.

2-Oxo-3-phenyl-1-oxaspiro[4.5]dec-3-en-4-yl methanesulfonate (6s): This compound was obtained following the abovedescribed method as white powder, yield $92 \%, \operatorname{mp~} 112-113{ }^{\circ} \mathrm{C}$; ${ }^{1} \mathrm{H} \mathrm{NMR}\left(400 \mathrm{MHz}, \mathrm{CDCl}_{3}\right) \delta 7.50$ (d, J=6.8 Hz, 2H, Ph-H), 7.35-7.40 (m, 3H, Ph-H), 2.89 (s, 3H, $\left.\mathrm{CH}_{3}\right), 1.17-1.94$ (m, $10 \mathrm{H}$, Cyclohexyl-H); ${ }^{13} \mathrm{C} \mathrm{NMR}\left(100 \mathrm{MHz}, \mathrm{CDCl}_{3}\right) \delta 169.95$, 169.38, 130.65, 130.02, 129.75, 128.37, 119.51, 85.23, 41.92, 33.68, 25.21, 22.69; ESI-MS: calcd for $\mathrm{C}_{16} \mathrm{H}_{18} \mathrm{O}_{5} \mathrm{~S}\left([\mathrm{M}+1]^{+}\right)$, 323.4; found, 323.8 .

Ethyl 2-oxo-3-phenyl-1-oxaspiro[4.5]dec-3-en-4-yl carbonate (6t): This compound was obtained following the abovedescribed method as light yellow powder, yield 93\%, mp 71 $72{ }^{\circ} \mathrm{C} ;{ }^{1} \mathrm{H}$ NMR $\left(400 \mathrm{MHz}, \mathrm{CDCl}_{3}\right) \delta 7.55(\mathrm{~d}, J=7.6 \mathrm{~Hz}, 2 \mathrm{H}$, $\mathrm{Ph}-\mathrm{H}$ ), 7.25-7.35 (m, 3H, Ph-H), 4.07 (q, $J=7.2 \mathrm{~Hz}, 2 \mathrm{H}, \mathrm{CH}_{2}$ $\mathrm{CH}_{3}$ ), 1.66-1.79 (m, 10H, Cyclohexyl-H), 1.12 (t, $J=7.2 \mathrm{~Hz}$, $\left.3 \mathrm{H}, \mathrm{CH}_{2} \mathrm{CH}_{3}\right) ;{ }^{13} \mathrm{C} \mathrm{NMR}\left(100 \mathrm{MHz}, \mathrm{CDCl}_{3}\right) \delta 169.58,169.27$, 149.10, 128.96, 128.62, 128.07, 127.73, 114.70, 83.14, 66.30, 32.73, 24.34, 21.68, 13.84; ESI-MS: calcd for $\mathrm{C}_{18} \mathrm{H}_{20} \mathrm{O}_{5}([\mathrm{M}+$ $\left.1]^{+}\right), 317.3$; found, 371.9 .

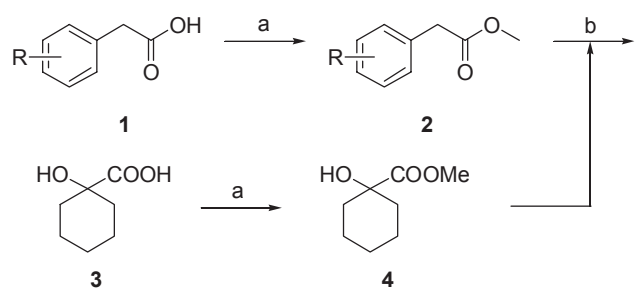

Biology assay. All bioassays were performed on representative test organisms reared in the laboratory. The bioassay was repeated at $25 \pm 1{ }^{\circ} \mathrm{C}$ according to statistical requirements. All compounds were dissolved in EtOH (AP, Shanghai Chemical Reagent Co., Ltd., Shanghai, China) and diluted with distilled water containing Triton X-100 $(0.1 \mu \mathrm{g} / \mathrm{mL})$ to obtain series concentrations of 1000,333 , and $111 \mu \mathrm{g} / \mathrm{mL}$ and others for bioassays. For comparative purposes, spirodiclofen was tested under the same conditions.

\section{Results and Discussion}

Synthesis of substituted spiro-tetronic acid derivatives 6a-t. Considering the convenient synthesis to construct the spirocycle scaffold, spirodiclofen analogues 6a-t was prepared in a new and simple route, which is outlined in Scheme 1.

As shown in Scheme 1, the target compounds were conveniently obtained via three steps including esterification, heterocyclization and acylation reactions. The key intermediate 3-(substituted phenyl)-4-hydroxy- $\Delta^{3}$-dihydrofuran-2-one 5a-d was accessed conveniently by the modified one-pot heterocyclization reaction ${ }^{27,28}$ from various substituted methyl 2-phenylacetate 2 and methyl 1-hydroxycyclohexanecarboxylate 4 in $70-83 \%$ yield, which involve a transesterification step, followed by the Dieckmann condensation. According the above-described experimental conditions, the key intermediates 5a-d were achieved with a high yield and simple disposal compared the reported cyclization methods. ${ }^{27,28}$ Meanwhile, this synthetic route leads to a convenient access to spirodiclofen analogues compared the reported traditional methods, ${ }^{29-34}$ which may be propitious to large scale production. The structures of all newly synthesized compounds were characterized as 3-(substituted-phenyl)-1oxaspiro[4.5]dec-3-en-2-one derivatives 6a-t on the basis of satisfactory analytical and spectral data including ${ }^{1} \mathrm{H}$ NMR, ${ }^{13} \mathrm{C}$ NMR and ESI-MS.

Biological activity evaluation. The bioactivity of synthesized spirodiclofen analogues against Aphid, Plutella xylostella, Cotton bollworm were evaluated according to a slightly modified FAO dip test, ${ }^{35}$ and the herbicidal activities of compounds 6a-t against Cynodon dactylon L., Lemna minor Linn, Brassica chinensis were tested using a previously reported procedure. ${ }^{36}$ All the insecticidal and herbicidal activities of synthesized spirotetronic acid derivatives 6a-t are listed in Table 1 and 2, respectively.

As we can see from Table 1, the preliminary assay showed that some of target molecules (such as compounds $\mathbf{6 a}, \mathbf{6 b}, \mathbf{6 c}$,

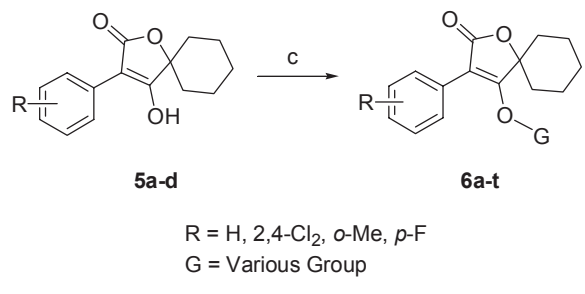

Scheme 1. Reagents and conditions: a. $\mathrm{MeOH}$, Conc. $\mathrm{H}_{2} \mathrm{SO}_{4}$, reflux $6-8$ h; b. EtONa or ${ }^{t} \mathrm{BuOK}$, THF, reflux for $8-10$ h; c. $\mathrm{GCl}_{2} \mathrm{Et}_{3} \mathrm{~N}, \mathrm{CH}_{2} \mathrm{Cl}_{2}$, $\mathrm{rt}$ for $0.5-4 \mathrm{~h}$ 
Table 1. Effects of substitution on insecticidal activity for compounds 6a-t at different concentration $(\mu \mathrm{g} / \mathrm{mL})$

\begin{tabular}{|c|c|c|c|c|c|c|c|c|c|c|c|c|}
\hline \multirow{2}{*}{ Entry } & \multirow{2}{*}{$\begin{array}{c}\text { Compd. } \\
\text { No. }\end{array}$} & \multicolumn{2}{|r|}{ Substituents } & \multicolumn{3}{|c|}{$\begin{array}{l}\text { Efficacy against } \\
\text { Aphid }\end{array}$} & \multicolumn{3}{|c|}{$\begin{array}{c}\text { Efficacy against } \\
\text { Plutella xylostella }\end{array}$} & \multicolumn{3}{|c|}{$\begin{array}{l}\text { Efficacy against } \\
\text { Cotton bollworm }\end{array}$} \\
\hline & & $\mathrm{R}$ & G & 1000 & 333 & 111 & 1000 & 333 & 111 & 1000 & 333 & 111 \\
\hline 1 & $6 \mathbf{a}$ & $2,4-\mathrm{Cl}_{2}$ & $\mathrm{SO}_{2} \mathrm{Me}$ & 0 & 0 & 0 & 9 & 9 & 9 & 0 & 0 & 0 \\
\hline 2 & $6 b$ & $2,4-\mathrm{Cl}_{2}$ & COOEt & 0 & 0 & 0 & 9 & 9 & 9 & 0 & 0 & 0 \\
\hline 3 & $6 c$ & $2,4-\mathrm{Cl}_{2}$ & $\mathrm{COCH}_{2}-\mathrm{C}_{6} \mathrm{H}_{5}$ & 0 & 0 & 0 & 9 & 9 & 9 & 0 & 0 & 0 \\
\hline 4 & 6d & $2,4-\mathrm{Cl}_{2}$ & $\mathrm{COCH}_{2}-2,4-\mathrm{Cl}_{2}-\mathrm{C}_{6} \mathrm{H}_{3}$ & 0 & 0 & 0 & 9 & 9 & 9 & 0 & 0 & 0 \\
\hline 5 & $6 e$ & $2,4-\mathrm{Cl}_{2}$ & $\mathrm{COCH}_{2}-2-\mathrm{Me}-\mathrm{C}_{6} \mathrm{H}_{4}$ & 0 & 0 & 0 & 9 & 9 & 9 & 0 & 0 & 0 \\
\hline 6 & $6 f$ & $2,4-\mathrm{Cl}_{2}$ & $\mathrm{CO}-3,4,5-(\mathrm{MeO})_{3}-\mathrm{C}_{6} \mathrm{H}_{2}$ & 0 & 0 & 0 & 9 & 0 & 0 & 0 & 0 & 0 \\
\hline 7 & $6 \mathrm{~g}$ & $2,4-\mathrm{Cl}_{2}$ & $\mathrm{CO}-4-\mathrm{F}-\mathrm{C}_{6} \mathrm{H}_{4}$ & 0 & 0 & 0 & 5 & 5 & 0 & 0 & 0 & 0 \\
\hline 8 & $6 \mathrm{~h}$ & $o-\mathrm{Me}$ & $\mathrm{COCMe}_{2} \mathrm{Et}$ & 0 & 0 & 0 & 5 & 5 & 5 & 0 & 0 & 0 \\
\hline 9 & $6 \mathbf{i}$ & $o-\mathrm{Me}$ & $\mathrm{SO}_{2} \mathrm{Me}$ & 0 & 0 & 0 & 9 & 9 & 9 & 0 & 0 & 0 \\
\hline 10 & $6 \mathbf{j}$ & $o-\mathrm{Me}$ & COOEt & 0 & 0 & 0 & 9 & 9 & 9 & 9 & 0 & 0 \\
\hline 11 & $6 \mathbf{k}$ & $o-\mathrm{Me}$ & $\mathrm{COCH}_{2}-2,4-\mathrm{Cl}_{2}-\mathrm{C}_{6} \mathrm{H}_{3}$ & 0 & 0 & 0 & 5 & 5 & 5 & 0 & 0 & 0 \\
\hline 12 & 61 & $o-\mathrm{Me}$ & $\mathrm{COCH}_{2}-2-\mathrm{Me}-\mathrm{C}_{6} \mathrm{H}_{4}$ & 0 & 0 & 0 & 9 & 9 & 9 & 0 & 0 & 0 \\
\hline 13 & $6 \mathrm{~m}$ & $o-\mathrm{Me}$ & $\mathrm{CO}-3,4,5-(\mathrm{MeO})_{3}-\mathrm{C}_{6} \mathrm{H}_{2}$ & 0 & 0 & 0 & 0 & 0 & 0 & 0 & 0 & 0 \\
\hline 14 & $6 n$ & $o-\mathrm{Me}$ & $\mathrm{CO}-4-\mathrm{F}-\mathrm{C}_{6} \mathrm{H}_{4}$ & 0 & 0 & 0 & 5 & 5 & 5 & 0 & 0 & 0 \\
\hline 15 & 60 & $4-\mathrm{F}$ & $\mathrm{COCMe}_{2} \mathrm{Et}$ & 0 & 0 & 0 & 0 & 0 & 0 & 0 & 0 & 0 \\
\hline 16 & $6 p$ & $4-\mathrm{F}$ & $\mathrm{SO}_{2} \mathrm{Me}$ & 0 & 0 & 0 & 0 & 0 & 0 & 0 & 0 & 0 \\
\hline 17 & $6 q$ & $4-\mathrm{F}$ & COOEt & 0 & 0 & 0 & 0 & 0 & 0 & 0 & 0 & 0 \\
\hline 18 & $6 r$ & $\mathrm{H}$ & $\mathrm{COCMe}_{2} \mathrm{Et}$ & 0 & 0 & 0 & 0 & 0 & 0 & 0 & 0 & 0 \\
\hline 19 & $6 s$ & $\mathrm{H}$ & $\mathrm{SO}_{2} \mathrm{Me}$ & 0 & 0 & 0 & 0 & 0 & 0 & 0 & 0 & 0 \\
\hline 20 & $6 \mathrm{t}$ & $\mathrm{H}$ & COOEt & 0 & 0 & 0 & 0 & 0 & 0 & 0 & 0 & 0 \\
\hline 21 & \multicolumn{3}{|c|}{ Spirodiclofen } & 0 & 0 & 0 & 9 & 9 & 9 & 0 & 0 & 0 \\
\hline
\end{tabular}

Scale: 0 (Weak, 0 - 40\% mortality); 5 (Moderate, 41 - 80\% mortality); 9 (Very good, 81 - 100\% mortality).

6d, 6e, 6i, 6j, and 6l) displayed obviously good and selective insecticidal activity against Plutella xylostella compared with spirodiclofen (at low concentration of $111 \mu \mathrm{g} / \mathrm{mL}$, Table 1), but presented low activity against Aphid and Cotton bollworm. In addition, we introduced electron-withdrawing groups (such as halogen and fluorine atoms) and electron-donating substituents $(\mathrm{Me})$ into the aromatic ring for exploring the influence of structural changes on activity. As described in Table 1, the different substituent at the periphery of the molecules 6a-t can lead to the obviously different inhibition activities. Compounds containing 2,4-dichloro and ortho-methyl substituents 6a-n and compounds bearing 4-fluoro and hydrogen substituents 60-t show a striking contrast, the latter almost lost activities at the same concentration level (Entry 15-20, Table 1), which may be indicated position changes of the substituent within the aromatic ring dramatically affect the activity, suggesting that the presence of a group in the ortho-position of aromatic ring could introduce important steric and electronic effects.

Additionally, we can find from Table 1 that the insecticidal activities of the target compounds 6a-t were also influenced by the different moiety attached to the oxygen atom of keto-enol cycle. As shown in Table 1, the function groups $\mathrm{G}$ are $\mathrm{SO}_{2} \mathrm{Me}$ and COOEt, which are more favorable for insecticidal activities. However, when the groups $\mathrm{G}$ are substituted-benzoyl such as compounds $6 \mathbf{6}$ and $\mathbf{6 g}, \mathbf{6 m}$ and $\mathbf{6 n}$, decreases the potency.

From Table 2, which shows the structures and the herbicidal activity data, it can be seen that most of the synthesized spirodiclofen analogues 6a-t showed good and selective efficacy against Brassica chinensis in higher concentrations $\left(1.0 \times 10^{-3}\right)$ except compounds $\mathbf{6 f}, \mathbf{6 j}, \mathbf{6} \mathbf{k}$, and $\mathbf{6 m}$. It is note worthy that most of target compounds including spirodiclofen exhibited low activity against Lemna minor Linn. and Cynodon dactylon L. As we know, Lemna minor Linn. and Cynodon dactylon L. are two difficult species in weed control. However, only the synthesized spirocyclic analogues $\mathbf{6 j}$ revealed obviously better herbicidal performance against these two target species compared with commercial spirodiclofen, which indicated that compound $\mathbf{6 j}$ might be used to further optimize for development novel weeds control agents.

The structure evolution here was to replace moiety A and moiety B, respectively (Figure 3). According to the results shown in Table 1, we can obtain the preliminary structure-activity profile for these spirocyclic tetronic acid analogues. As indicated in Figure 3, for moiety A, the compounds bearing 2,4-Cl 2 -phenyl and 2-Me-phenyl substituents such as 6a-n show better activities, however, when the substituents $\mathrm{R}$ are $\mathrm{H}$ and 4-F (such as 6o-t), which present low efficacy. Moiety B was varied among such substituents as -COOEt, $-\mathrm{SO}_{2} \mathrm{Me},-\mathrm{COCMe}_{2} \mathrm{Et}$, substi- 
Table 2. Effects of substitution on herbicidal activity for compounds 6a-t at different concentration $(\mu \mathrm{g} / \mathrm{mL})$

\begin{tabular}{|c|c|c|c|c|c|c|c|c|c|c|c|c|}
\hline \multirow{2}{*}{ Entry } & \multirow{2}{*}{$\begin{array}{l}\text { Compd. } \\
\text { No. }\end{array}$} & \multicolumn{2}{|r|}{ Substituents } & \multicolumn{3}{|c|}{$\begin{array}{l}\text { Efficacy against } \\
\text { Lemna minor Linn. }\end{array}$} & \multicolumn{3}{|c|}{$\begin{array}{c}\text { Efficacy against } \\
\text { Cynodon dactylon } \mathrm{L} .\end{array}$} & \multicolumn{3}{|c|}{$\begin{array}{c}\text { Efficacy against } \\
\text { Brassica chinensis. }\end{array}$} \\
\hline & & $\mathrm{R}$ & G & 1000 & 333 & 111 & 1000 & 333 & 111 & 1000 & 333 & 111 \\
\hline 1 & 6a & $2,4-\mathrm{Cl}_{2}$ & $\mathrm{SO}_{2} \mathrm{Me}$ & 0 & 0 & 0 & 0 & 0 & 0 & 9 & 0 & 0 \\
\hline 2 & $6 b$ & $2,4-\mathrm{Cl}_{2}$ & COOEt & 0 & 0 & 0 & 0 & 0 & 0 & 9 & 0 & 0 \\
\hline 3 & $6 c$ & $2,4-\mathrm{Cl}_{2}$ & $\mathrm{COCH}_{2}-\mathrm{C}_{6} \mathrm{H}_{5}$ & 0 & 0 & 0 & 0 & 0 & 0 & 9 & 0 & 0 \\
\hline 4 & 6d & $2,4-\mathrm{Cl}_{2}$ & $\mathrm{COCH}_{2}-2,4-\mathrm{Cl}_{2}-\mathrm{C}_{6} \mathrm{H}_{3}$ & 0 & 0 & 0 & 0 & 0 & 0 & 9 & 0 & 0 \\
\hline 5 & $6 e$ & $2,4-\mathrm{Cl}_{2}$ & $\mathrm{COCH}_{2}-2-\mathrm{Me}-\mathrm{C}_{6} \mathrm{H}_{4}$ & 0 & 0 & 0 & 0 & 0 & 0 & 9 & 0 & 0 \\
\hline 6 & $6 f$ & $2,4-\mathrm{Cl}_{2}$ & $\mathrm{CO}-3,4,5-(\mathrm{MeO})_{3}-\mathrm{C}_{6} \mathrm{H}_{2}$ & 0 & 0 & 0 & 0 & 0 & 0 & 0 & 0 & 0 \\
\hline 7 & $6 \mathrm{~g}$ & $2,4-\mathrm{Cl}_{2}$ & $\mathrm{CO}-4-\mathrm{F}-\mathrm{C}_{6} \mathrm{H}_{4}$ & 0 & 0 & 0 & 0 & 0 & 0 & 9 & 0 & 0 \\
\hline 8 & $6 \mathrm{~h}$ & $o-\mathrm{Me}$ & $\mathrm{COCMe}_{2} \mathrm{Et}$ & 0 & 0 & 0 & 0 & 0 & 0 & 9 & 0 & 0 \\
\hline 9 & $6 \mathbf{i}$ & $o-\mathrm{Me}$ & $\mathrm{SO}_{2} \mathrm{Me}$ & 0 & 0 & 0 & 0 & 0 & 0 & 9 & 0 & 0 \\
\hline 10 & $6 \mathbf{j}$ & $o-\mathrm{Me}$ & COOEt & 9 & 9 & 0 & 9 & 9 & 0 & 0 & 0 & 0 \\
\hline 11 & $6 \mathbf{k}$ & $o-\mathrm{Me}$ & $\mathrm{COCH}_{2}-2,4-\mathrm{Cl}_{2}-\mathrm{C}_{6} \mathrm{H}_{3}$ & 0 & 0 & 0 & 0 & 0 & 0 & 0 & 0 & 0 \\
\hline 12 & 61 & $o-\mathrm{Me}$ & $\mathrm{COCH}_{2}-2-\mathrm{Me}-\mathrm{C}_{6} \mathrm{H}_{4}$ & 0 & 0 & 0 & 0 & 0 & 0 & 9 & 0 & 0 \\
\hline 13 & $6 \mathrm{~m}$ & $o-\mathrm{Me}$ & $\mathrm{CO}-3,4,5-(\mathrm{MeO})_{3}-\mathrm{C}_{6} \mathrm{H}_{2}$ & 0 & 0 & 0 & 0 & 0 & 0 & 0 & 0 & 0 \\
\hline 14 & 6n & $o-\mathrm{Me}$ & $\mathrm{CO}-4-\mathrm{F}-\mathrm{C}_{6} \mathrm{H}_{4}$ & 0 & 0 & 0 & 0 & 0 & 0 & 9 & 0 & 0 \\
\hline 15 & 60 & $4-\mathrm{F}$ & $\mathrm{COCMe}_{2} \mathrm{Et}$ & 0 & 0 & 0 & 0 & 0 & 0 & 9 & 0 & 0 \\
\hline 16 & $6 p$ & $4-\mathrm{F}$ & $\mathrm{SO}_{2} \mathrm{Me}$ & 0 & 0 & 0 & 0 & 0 & 0 & 9 & 0 & 0 \\
\hline 17 & $6 q$ & $4-\mathrm{F}$ & COOEt & 0 & 0 & 0 & 0 & 0 & 0 & 9 & 0 & 0 \\
\hline 18 & $6 r$ & $\mathrm{H}$ & $\mathrm{COCMe}_{2} \mathrm{Et}$ & 0 & 0 & 0 & 0 & 0 & 0 & 0 & 0 & 0 \\
\hline 19 & $6 s$ & $\mathrm{H}$ & $\mathrm{SO}_{2} \mathrm{Me}$ & 0 & 0 & 0 & 0 & 0 & 0 & 9 & 0 & 0 \\
\hline 20 & $6 t$ & $\mathrm{H}$ & COOEt & 9 & 0 & 0 & 0 & 0 & 0 & 9 & 0 & 0 \\
\hline 21 & \multicolumn{3}{|c|}{ Spirodiclofen } & 0 & 0 & 0 & 0 & 0 & 0 & 9 & 0 & 0 \\
\hline
\end{tabular}

Scale: 0 (Weak, 0 - 40\% mortality); 5 (Moderate, 41 - 80\% mortality); 9 (Very good, 81 - 100\% mortality).

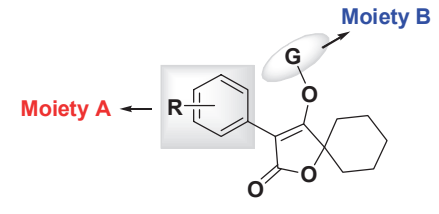

Analogues of Spirodiclofen
Activity order of Moiety A: $\mathrm{R}=2,4-\mathrm{Cl}_{2}>\mathrm{R}=\mathrm{O}-\mathrm{Me}>\mathrm{R}=\mathrm{H}$ and $\mathrm{R}=4-\mathrm{F}$

Activity order of Moiety B: $-\mathrm{COOEt}-\mathrm{SO}_{2} \mathrm{Me}-\mathrm{COCMe}_{2} \mathrm{Et}>-\mathrm{COCH}_{2}-2-\mathrm{Me}_{-} \mathrm{C}_{6} \mathrm{H}_{4}$

$-\mathrm{COCH}_{2}-2,4-\mathrm{Cl}_{2}-\mathrm{C}_{6} \mathrm{H}_{3}-\mathrm{COCH}_{2}-\mathrm{C}_{6} \mathrm{H}_{5}>-\mathrm{CO}-3,4,5-(\mathrm{MeO})_{3}-\mathrm{C}_{6} \mathrm{H}_{2} \quad-\mathrm{CO}-4-\mathrm{F}-\mathrm{C}_{6} \mathrm{H}_{4}$

Figure 3. General structure-activity profile for the spirodiclofen analogues.

tuted phenylacetyl and substituted benzoyl, the results testified that compounds containing -COOEt, $-\mathrm{SO}_{2} \mathrm{Me}$, and $-\mathrm{COCMe} \mathrm{E}_{2} \mathrm{Et}$ group exhibited obviously high activity than compounds bearing substituted benzoyl moiety, which may be due to the steric size of phenyl is unfavourable for the binding to receptor.

\section{Conclusion}

In summary, we have described the convenient synthesis, biological activities, and SAR of a series of spirodiclofen analogues, and developed a practical and efficient procedure for the large scale synthesis of spiro-tetronic acid derivatives through the direct one-pot heterocyclization reaction of methyl 1-hydroxycyclohexanecarboxylate with various esters in THF. According the above-described experimental conditions, the cyclization reaction reached completion with a very high yield and simple disposal. The preliminary bioassay results indicated that some of the analogues exhibited good insecticidal and herbicidal activity compared to commercialized spirodiclofen. Furthermore, the understanding of structure-activity relationship (SAR) may be advantageous for further structure optimization and thereby provides some insight into the rational design of novel inhibitors targeting lipid biosynthesis.

Acknowledgments. This work was financially supported by the Hubei Biopesticide Engineering Research Centre. The authors also gratefully acknowledge the partial support from Hubei Agricultural Science Innovation Centre (2007-620-001$03)$. The authors also would like to thank many colleagues of the Hubei Biopesticide Engineering Research Centre and the Hubei Academy of Agricultural Sciences for valuable advice and assistance. 


\section{References}

1. Wing, K. D.; Sacher, M.; Kagaya, Y.; Tsurubuchi, Y.; Mulderig, L.; Connair, M.; Schnee, M. Crop Prot. 2000, 19, 537.

2. Qian, X.; Lee, P. W.; Cao, S. J. Agric. Food Chem. 2010, 58, 2613.

3. Suzuki, J.; Ishida, T.; Kikuchi, Y.; Ito, Y.; Morikawa, C.; Tsukidate, Y.; Tanji, I.; Ota, Y.; Toda, K. J. Pestic. Sci. 2002, 27, 1.

4. Fischer, R.; Bretschneider, T.; Krueger, B. W.; Bachmann, J.; Erdelen, C.; Wachendorff-Neumann, U.; Santel, H. J.; Luerssen, K.; Schmidt, R. R. DE 4216814, 1993.

5. Wachendorff, U.; Brück, E.; Elbert, A.; Fischer, R.; Nauen, R.; Stumpf, N.; Tiemann, R. Proc. Brighton Crop Protection Conference-Pests \& Diseases 2000, 2A-6, 53.

6. Fischer, R.; Benet-Buchholz, J. Pflanzenschutz-Nachrichten Bayer 2002, 55, 137.

7. Wachendorff, U.; Nauen, R.; Schnorbach, H. J.; Rauch, N.; Elbert, A. Pflanzenschutz-Nachrichten Bayer 2002, 55, 149.

8. Chang, J.-Y.; Lu, K.-H.; Hsieh, T.-T. Plant Prot. Bull. 2007, 49, 187.

9. Bretschneider, T.; Fischer, R.; Nauen, R. In Modern Crop Protection Compounds; Krämer, W., Schirmer, U. Eds.; Wiley-VCH Verlag GmbH \& Co: Weinheim, Germany, pp 909-925, 2007.

10. Nauen, R.; Bretschneider, T.; Elbert, A.; Fisher, R.; Tiemann, R. Pestic. Outlook 2003, 12, 243.

11. Dekeyser, M. A. Pest Manag. Sci. 2005, 61, 103.

12. Bretschneider, T.; Benet-Buchholz, J.; Fischer, R.; Nauen, R. Chimia 2003, 57, 697.

13. Bretschneider, T.; Fischer, R.; Benet-Buchholz, J. PflanzenschutzNachrichten Bayer 2005, 58, 307.

14. Nauen, R.; Bretschneider, T.; Brueck, E.; Elbert, A.; Reckmann, U.; Wachendorff, U.; Tiemann, R. Proc. Brighton Crop Protection Conference-Pests \& Diseases 2002, 2A-3, 39.

15. Liu, T.-X. Crop Prot. 2004, 23, 505.

16. Bielza, P.; Fernández, E.; Grávalos, C.; Izquierdo, J. Bio Control 2009, 54, 229.

17. Fischer, R.; Himmler, T.; Nauen, R.; Reckmann, U.; Schmitt, W. Int. Plant Prot. Congress (IPPC), Glasgow, 2007; p 100.

18. Van Waetermeulen, X.; Brück, E.; Elbert, A.; Fischer, R.; Krueger, S.; Kühnhold, J.; Nauen, R.; Niebes, J. F.; Reckmann, U.; Schnor- bach, H. J.; Steffens, R. Int. Plant Prot. Congress (IPPC), Glasgow, 2007; p 60 .

19. Nauen, R.; Bretschneider, T.; Elbert, A.; Fischer, R.; Reckmann, U.; van Waetermeulen, X. 11th IUPAC International Congress of Pesticide Chemistry, Aug 6-11. Kobe, Japan, 2006; p 109.

20. Fischer, R.; Wei, H.-C. Bayer CropScience J. 2008, 61, 127.

21. Brück, E.; Elbert, A.; Fischer, R.; Krueger, S.; Kühnhold, J.; Klueken, A. M.; Nauen, R.; Niebes, J. F.; Reckmann, U.; Schnorbach, H. J.; Steffens, R.; van Waetermeulen, X. Crop Prot. 2009, 28, 838.

22. Van Pottelberge, S.; Van Leeuwen, T.; Khajehali, J.; Tirry, L. Pest Manag. Sci. 2009, 65, 358.

23. Nauen, R.; Stumpf, N.; Elbert, A. Proc. Brighton Crop Protection Conference - Pests \& Diseases 2000, 4D-9, 453.

24. Rauch, N.; Nauen, R. Pestic. Biochem. Phys. 2003, 74, 91.

25. Nauen, R. J. Pestic. Sci. 2005, 30, 272.

26. Van Pottelberge, S.; Khajehali, J.; Van Leeuwen, T.; Tirry, L. Exp. Appl. Acarol. 2009, 47, 301.

27. Mallinger, A.; Le Gall, T.; Mioskowski, C. Synlett 2008, 386.

28. Mallinger, A.; Le Gall, T.; Mioskowski, C. J. Org. Chem. 2009, 74,1124 .

29. Fischer, R.; Bretschneider, T.; Krüger, B. W.; Bachmann, J.; Erdelen, C.; Wachendorff-Neumann, U.; Santel, H. J.; Lürssen, K.; Schmidt, R. R. US 5262383, 1993.

30. Fischer, R.; Lehr, S.; Feucht, D.; Lösel, P.; Malsam, O.; Bojack, G.; Auler, T.; Hills, M. J.; Kehne, H.; Rosinger, C. H. WO 2005066125 , 2005.

31. Fischer, R.; Lehr, S.; Feucht, D.; Lösel, P.; Malsam, O.; Bojack, G.; Auler, T.; Hills, M. J.; Kehne, H.; Rosinger, C. H. US 20070244007 , 2007.

32. Lu, Y.; Xu, X.; Sun, N.; Zhao, J. Fine Chem. Intermed. 2009, 39, 19.

33. Zhao, J.; Ji, M.; Xu, X.; Cheng, J.; Zhu, G. Chin. Chem. Lett. 2009, $20,1307$.

34. Zhao, J.; Zhou, Y.; Xu, X.; Cheng, J.; Zhu, G. Chin. J. Struct. Chem. 2009, 28, 837.

35. FAO. Recommended methods for the detection and measurement of resistance of agricultural pests to pesticides: method for adult aphids; FAO method 17. FAO Plant Prot. Bull. 1979, 18, 6.

36. Xue, S.; Ke, S.; Yan, L.; Cai, Z.; Wei, Y. J. Inorg. Biochem. 2005 , 99, 2276. 